\title{
The Anticholinesterase Phenserine and Its Enantiomer Posiphen as 5'Untranslated-Region-Directed Translation Blockers of the Parkinson's Alpha Synuclein Expression
}

\author{
Sohan Mikkilineni, ${ }^{1}$ Ippolita Cantuti-Castelvetri, ${ }^{2}$ Catherine M. Cahill, ${ }^{1}$ Amelie Balliedier, ${ }^{1}$ \\ Nigel H. Greig, ${ }^{3}$ and Jack T. Rogers ${ }^{1}$ \\ ${ }^{1}$ Neurochemistry Laboratory, Massachusetts General Hospital (East), CNY2, 149, 13th Street, Charlestown, MA 02129, USA \\ ${ }^{2}$ MIND, Massachusetts General Hospital, Charlestown, MA 02129, USA \\ ${ }^{3}$ Drug Design and Development Section, Laboratory of Neurosciences, Intramural Research Program, National Institute on Aging, \\ Baltimore, MD 21224, USA
}

Correspondence should be addressed to Jack T. Rogers, jrogers@partners.org

Received 1 July 2011; Accepted 29 February 2012

Academic Editor: Paul S. Foster

Copyright ( 2012 Sohan Mikkilineni et al. This is an open access article distributed under the Creative Commons Attribution License, which permits unrestricted use, distribution, and reproduction in any medium, provided the original work is properly cited.

There is compelling support for limiting expression of alpha-synuclein $(\alpha-s y n)$ in the brains of Parkinson's disease (PD) patients. An increase of SNCA gene copy number can genetically cause familial PD where increased dose of this pathogenic protein correlates with severity of symptoms (triplication of the SNCA gene causes dementia in PD patients). Gene promoter polymorphisms were shown to increase $\alpha$-synuclein expression as a risk for PD. Cholinesterase inhibitors can clinically slow cognitive decline in the later stages of PD etiology similar to their widespread use in Alzheimer's disease (AD). Pertinent to this, we identified that the well-tolerated anticholinesterase, phenserine, blocked neural SNCA mRNA translation and tested for targeting via its $5^{\prime}$ untranslated region ( $\left.5^{\prime} \mathrm{UTR}\right)$ in a manner similar to its action to limit the expression of the AD-specific amyloid precursor protein (APP). Posiphen, its better-tolerated $(+)$ enantiomer (devoid of anticholinesterase action), repressed neural $\alpha$-synuclein translation. Primary metabolic analogs of posiphen were, likewise, characterized using primary fetal neurons grown ex vivo from the brains of Parkinson's transgenic mice expressing the human SNCA gene.

\section{Introduction}

Parkinson disease (PD) is a slowly progressive neurodegenerative disease affecting up to $3 \%$ of the population over the age of 65 years. Clinically, it is characterized by a set of motor symptoms and nonmotor symptoms that can include dementia. Motor symptoms include rigidity, postural instability, bradykinesia, and resting tremor $[1,2]$. The core pathological feature correlating with most of these motor symptoms is a loss of dopaminergic neurons from the substantia nigra pars compacta (SNc). Pathological inclusions, known as Lewy bodies, are found within some of the remaining dopaminergic neurons [3]. The destruction of the dopaminergic neurons at onset is at least $70 \%$ and, at the end stage of the disease the loss of dopamine (DA) neurons, can exceed 95\% [3]. Therapy for the symptoms of PD is based primarily on replacement of dopaminergic function and can be remarkably effective in alleviating motor symptoms for a number of years $[1,4]$. Additional nonmotor symptoms can be present and include depression, anxiety, sensory abnormalities, anosmia, sleep, and autonomic disorders, in addition to dementia $[1,5-8]$.

Particular nonmotor symptoms, like sleep disturbances [9], loss of smell $[6,10]$, and depression, can occur well before the presence of detectable motor symptoms [5]. The presence of psychotic symptoms, which can affect up to $40 \%$ of the patients, is often associated with anti-parkinsonian 
treatment and is dose dependent $[2,7]$. By contrast, the neuropsychiatric symptoms of $\mathrm{PD}$, like depression and dementia, are more common at the later stages of the disease $[6,8$, $11,12]$. These neuropsychiatric symptoms may be correlated directly to the progression of PD itself as in PD dementia or PDD, to the presence of Dementia with Lewy Bodies (DLB) or to the presence of comorbidities like Alzheimer's Disease (PD/AD) [2]. The incidence of dementia in PD is six times higher than in age-matched controls; in all forms of dementia associated with PD, the use of cholinesterase inhibitors may be beneficial [2, 13-17].

The etiology of PD is still elusive [18]. A variety of genetic factors have been linked to the etiology of familial $\mathrm{PD}[19]$. The first was the protein alpha-synuclein $(\alpha-s y n)$, an abundant brain protein that appears to be involved in vesicle trafficking and participates in the regulation of DA release [20]. The first reports to implicate $\alpha$-syn in PD described mutations in the protein, which cause autosomal dominant forms of the disease $[21,22]$. Subsequent studies revealed that such mutations were exceedingly rare but also that aggregates of $\alpha$-syn could be found in all cases of familial and sporadic PD [23]. It is important to recognize that the genetic mechanisms identified to date are individually rare and, collectively, represent only a small fraction of the cases of PD observed by practitioners. This hence leaves the vast majority of PD unexplained at present.

Events associated with the inflammatory cascade [24] as well as with iron metabolism $[25,26]$ and translational control of gene expression have been modeled to be associated with PD and LBD [27]. There are also reported examples of disrupted signaling events such as occuring in response to inflammation cytokines: for example, mutations to the signaling kinase LRRK-2 may activate inflammatory events in this neurodegenerative disease $[24,28]$, perhaps affecting novel signalling pathways [24]. Certainly, increased iron in the individual dopaminergic neurons of the substantia nigra (SN) has been reported to be closely associated with the pathogenesis of PD [29], and antioxidants have been tested as a means to alleviate the severity of PD [30]. DLB brains exhibit lowered SNCA mRNA but higher insoluble protein, suggesting misregulation of SNCA mRNA translation additional to its clearance by chaperones [31], although reduced autophagy may provide the explanation. Translational control of $\alpha$-syn may be governed, at least in part, by a uniquely configured $5^{\prime}$ untranslated region ( $5^{\prime} \mathrm{UTR}$ ) of its transcript, which encodes a homology with the known APP and ferritin iron-responsive elements [32-35].

$\alpha$-Synuclein is, in fact, the central $\sim 15 \mathrm{kDa}$ protein implicated in the pathogenesis of neurodegenerative $\alpha$-synucleinopathies [36], including PD—-the most prevalent movement disorder in humans. Other alpha synucleinopathies include DLB, Lewy body variant of Alzheimer's disease (AD), and multiple system atrophy. In these disorders, $\alpha$ syn undergoes a conformational change and oligomerization, causing a toxic gain of function. Neurodegenerative deposition of $\alpha$-syn aggregates occurs, most commonly in Lewy bodies. Lewy bodies are rare in PD patients, present only in the surviving neurons. $\alpha$-syn aggregates are in dystrophic neurites. Glial cytoplasmic inclusions are in multiple systems atrophy [37]. Pathogenic pathways of $\alpha$-synuclein and amyloid- $\beta(A B)$ converge via interaction of these two amyloidogenic proteins, which coprecipitate into $B$-pleated oligomers and insoluble fibrils [38-40], although Aß and $\alpha$ syn rarely colocalize in brain amyloid deposits [41]. There is certainly also overlap between the 5'UTRs of the SNCA and amyloid precursor protein (APP) transcripts, another similarity that provides a further link between $\mathrm{PD}$ and $\mathrm{AD}$ [27, 33-35].

Such overlap in the $5^{\prime}$ UTRs of SNCA and APP mRNAs is consistent with our and other groups' recent demonstrations that both are key players in iron metabolism and $\alpha$-syn may be translationally controlled by cellular iron levels as was demonstrated for APP expression [35, 42]. In this regard, APP is a copper-dependent iron export ferroxidase [43], and $\alpha$-syn a Cu-binding ferrireductase [44, 45]. These similarities, together with the utility of anticholinesterases in $\mathrm{AD}$ and $\mathrm{PD}$, provided our rationale to test whether known anticholinesterases that are beneficial and $\mathrm{A} \beta$ lowering [46] would, likewise, provide anti- $\alpha$-syn activity in neural cells.

The most common therapy for PD is L-dopa that is routinely used to overcome the archetypical problems of tremor. As disease progresses, however, clinically available anticholinesterases have been increasingly empirically used, based in part on an putative impairment of the cholinergic system in developing PD [47].

Therefore, our study investigated the effectiveness of the cholinesterase inhibitor phenserine and its noncholinergic (+) chiral enantiomer, posiphen in lowering $\alpha$-synuclein expression in neural cell lines. Both agents have been shown to effectively lower APP synthesis rate both in neuronal cultures and animals via translational regulation mediated at the level of the APP mRNA 5'UTR. As both agents have been developed through to clinical studies and appear well tolerated in $\mathrm{AD}$, if effective in reducing $\alpha$-syn levels, they hold translational promise as potential therapeutics for PD.

Furthermore, as posiphen can be effectively dosed in rodents and humans in far greater amounts than phenserine, thereby generating high levels of its primary metabolites, the three most prominent primary metabolic products of posiphen were also characterized. As a model to examine the $\alpha$-syn-lowering efficacy of these agents, we assessed the cellular therapeutic impact of these screened SNCA 5 'UTR-directed translation blockers to reduce $\alpha$-synuclein expression in neural cells lines and subsequently also in the primary neurons from the $\mathrm{PAC} / \mathrm{Tg}(S N C A)$ genomic human SNCA mouse model of PD (Figures 2 and 6) [48].

1.1. Hypothesis/Model. Our model to be tested is shown in Figure 7. The overarching hypothesis is that the drug phenserine (anticholinesterase) and its enantiomer are known translation blockers of the amyloid precursor protein of Alzheimer's disease (AD) and, as such, have been tested in clinical trials for their antiamyloid efficacy and potential to improve cognition. We noted that the RNA target in the 5'UTR of APP mRNA was similar to that found in the alpha-synuclein transcript. Therefore, we decided to see if posiphen and phenserine might block $\alpha$-synuclein, 
the central culprit protein in PD. To achieve this end, we conducted Western Blot experiments firstly with SH-SY5Y dopaminergic neuroblastoma cells and then with primary E18 neurons from a genomic mouse model of PD. Our rationale for conducting this study was to determine if posiphen and phenserine are two $5^{\prime}$ UTR-directed drugs that would reduce alpha-synuclein expression to provide therapeutic benefit to Parkinson's disease patients.

\section{Materials and Methods}

2.1. Materials. Dulbecco's modified essential medium (DMEM, catalog no. 12-614Q); FBS (catalog no. 14-503E), L-glutamine (catalog no. 17-605E), penicillin/streptomycin (catalog no. 17-602E); phenol red free media (phenol redfree DMEM with $4.5 \mathrm{~g} / \mathrm{L}$ D-glucose (catalog no. 12917F)) were purchased from Lonza (Portsmouth, NH). Trypsin/EDTA (catalog no. 25-053-Cl) was purchased from Cellgro. Steady-Glo (catalog no. E2250) was purchased from Promega (Madison, WI). For the Western Blot secondary screen, penicillin/streptomycin was acquired from BioWhittaker (Walkersville, MD), mouse monoclonal antialpha-synuclein was purchased from BD Transduction Laboratories, and anti-beta-actin was acquired from Chemicon, Inc. We routinely use 384-well plates (catalog no. 3570), which were purchased from Corning and Falcon TC flasks (catalog no. 353112) were purchased from Becton Dickinson (Waltham, MA). SH-SY5Y cells were purchased from the ATCC, Manassas, VA.

2.2. Cell Culture. Transfected SH-SY5Y neuroblastoma cells were grown to confluency in $35 \mathrm{~mL}$ complete DMEM with $4.5 \mathrm{~g} / \mathrm{L}$ D-glucose supplemented with $10 \%$ FBS, $200 \mu \mathrm{M} \mathrm{L}$ glutamine, $100 \mu \mathrm{M}$ penicillin/streptomycin, and $200 \mu \mathrm{g} / \mathrm{mL}$ geneticin in a T175 TC flask in a TC incubator $\left(37^{\circ} \mathrm{C}\right.$, $95 \%$ humidity, $5 \% \mathrm{CO}_{2}$ ) (doubling time $=24 \mathrm{~h}$ ). Untransfected SH-SY5Y counterparts were grown in the absence of geneticin. Cells were harvested by washing the monolayer quickly with $5 \mathrm{~mL}$ trypsin/EDTA (1X), aspirating, then adding $5 \mathrm{~mL}$ trypsin/EDTA and incubating for $5 \mathrm{~min}$ at $37^{\circ} \mathrm{C}$, 95\% humidity, $5 \% \mathrm{CO}_{2}$, after which cells were collected into phosphate-buffered saline and centrifuged into a pellet for storage at $-70^{\circ} \mathrm{C}$.

Primary cortical neurons from wild-type mice and from the PAC-Tg(SNCA(wt) human SNCA genomic mice [48] were cultured as outlined by the method of Ray et al., 2009 [49]. Briefly, we recovered the embryonic day 1518 pups after sacrificing pregnant females, separated out the brain, and removed the meninges and blood vessels. We then dissected out the cortices and placed them in separate eppendorf tubes containing $500 \mathrm{uL}$ of HBSS without $\mathrm{Ca}^{+2} / \mathrm{Mg}^{+2}$ salts supplemented with $1 \mathrm{mM}$ sodium pyruvate and $10 \mathrm{mM}$ HEPES, $\mathrm{pH}$ 7.4. On ice, individual cells were isolated by titurating 10 times using a glass pasture pipette with the tip barely fire polished. We adjusted the volume to $1.5 \mathrm{~mL}$, by adding $1 \mathrm{~mL}$ of HBSS with $\mathrm{Ca}^{+2} / \mathrm{Mg}^{+2}$ salts $+\mathrm{Na}$ pyruvate + HEPES, restoring the divalent cations by adding HBSS so that the nondispersed tissue could settle for $5 \mathrm{~min}$, on ice. In the tissue culture laminar hood, we transferred the supernatant into a new $15 \mathrm{~mL}$ tube and centrifuged for $1 \mathrm{~min}$ at $900 \mathrm{rpm}, 4^{\circ} \mathrm{C}$. We gently resuspended the pellet in $2 \mathrm{~mL}$ of HBSS with $\mathrm{Ca}^{+2} / \mathrm{Mg}^{+2}$ salts + Na pyruvate + HEPES and took an aliquot for counting ( $2 \mathrm{~mL}$ for approx. 5 embryos). We then plated $\sim 1 \times 10^{5}$ cells/well of a 24 -well or $2 \times$ $10^{5} /$ in 12 -well plates. Each set of plates were coated with poly D-lysine containing poly L-lysine coverslips for micro immunocytochemical confirmation of neuronal integrity.

2.3. Bioinformatics Methods. The $\alpha$-synuclein RNA sequences were selected using the NCBI Gene search and the Ensembl database (see [34]). Since the $5^{\prime}$ UTRs were of primary interest, the coding sequences (CDS) were mostly disregarded, apart from the initiating AUG. As we reported [34], the splice junction between two exons occurred at a CAGUGU site $25=$ nucleotides from the AUG and that this pattern was conserved among the other species investigated. Thus, in order to study a balanced sequence, 25 nucleotides before the splice junction from the first exon were used to create 50 nucleotide RNA sequences (mouse and rat had 52 nt sequences due to insertions).

The ClustalX2 graphical program was used to align the RNA sequences to identify any evolutionary conservation between species. For alpha synuclein, the AUG start region of the CDS was a reference point for sequence alignments, allowing a comparison of the sequences in both exons around the splice junction, due to high local conservation fidelity. Secondary sequences were then generated by the University of Vienna's RNAfold webserver (with standard settings) and were annotated using the RNAfold command-line software. The RNAfold server provided the most probable secondary structure based on minimum-free energy calculations.

We calculated the alignment homology by comparing any species' RNA sequence (as listed) against the Homo sapiens sequence on each side of the splice junction. We also calculated the homology between the core L- and $\mathrm{H}$-ferritin IREs with that of alpha synuclein and APP mRNAs. Only nucleotides that matched respective to the Homo sapiens sequence were scored a point; we determined the percent homology on each side by totaling the points scored and dividing by the total number of nucleotides on that side. The results from each side were then compared to illuminate the difference in conservation across the splice junction.

2.4. Constructs. The SNCA-5'UTR-pGL3 construct was generated from the pGL3 expression vector (Promega). In this case, a PCR fragment encoding 48 base $\alpha$-synuclein $5^{\prime}$ UTR was cloned between the Hind-III and Ncol sites in front of start codon of the luciferase gene in PGL3. Transiently transfected cells expressed either pGL3 or the SNCA-5'UTRpGL3 construct. Stably transfected neural cell lines were generated via neomycin selection after cotransfection with the RSV2-neomycin plasmid to express the SNCA-5'UTRpGL3 construct (H2A cells) or pGL3 (control cells).

2.5. Stable-Transfection-Based Screen of a Library of Natural Product Inhibitors of $\alpha$-Synuclein Translation. A 720 compound natural products, including added phenserine and 


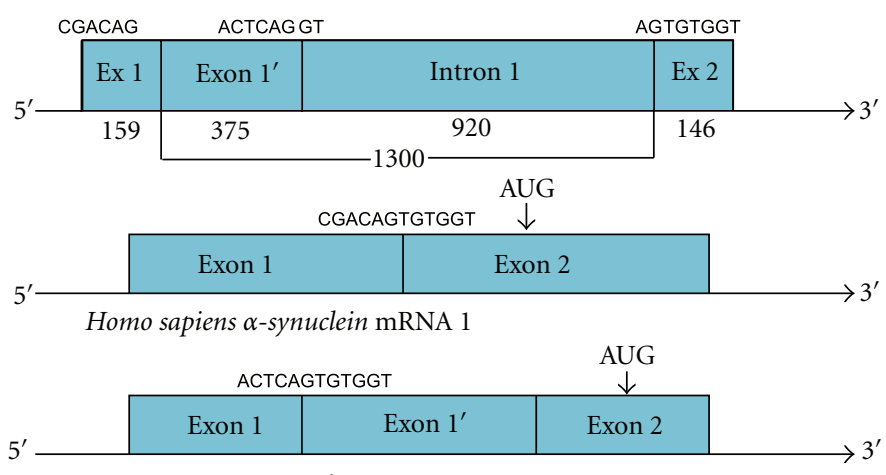

Homo sapiens $\alpha$-synuclein mRNA 2

(a)

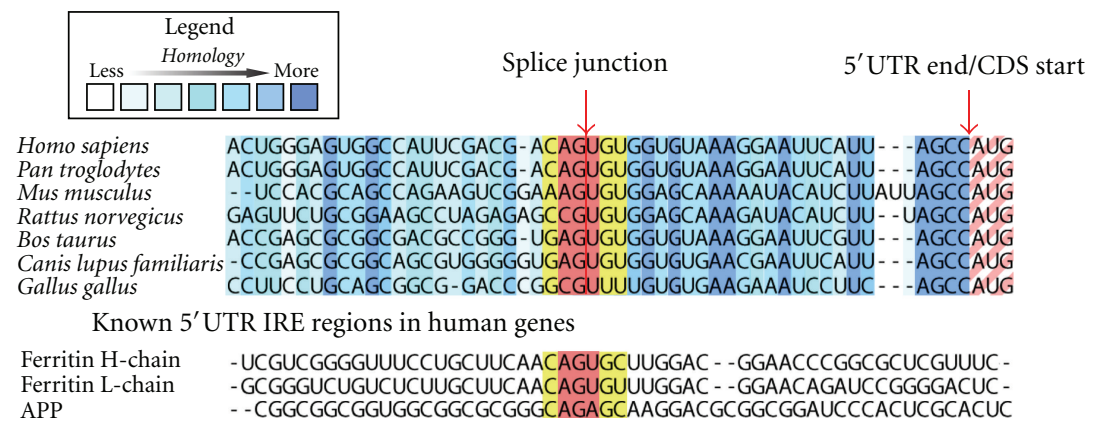

Evolutionary $\alpha$-synuclein RNA IRE structures
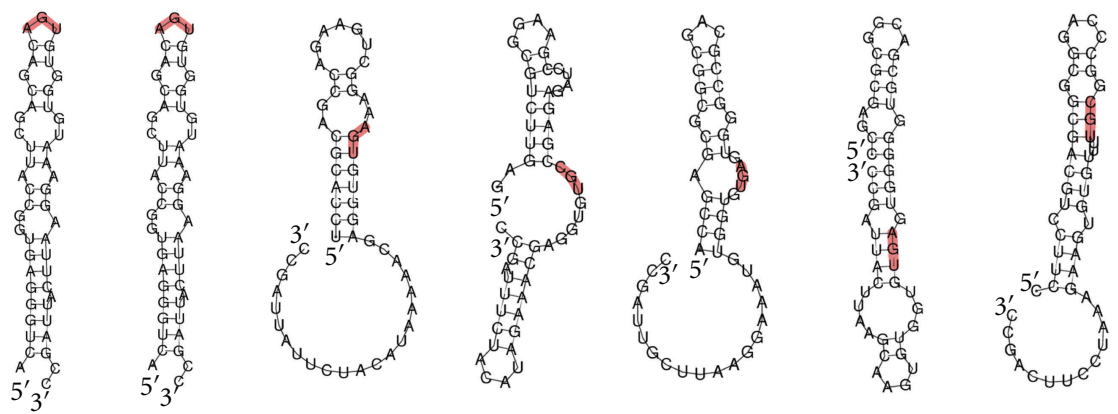

Homo sapiens Pan troglodytes Mus musculus $\alpha$-synuclein $\alpha$-synuclein $\alpha$-synuclein

\section{Rattus} norvegicus $\alpha$-synuclein
Bos taurus $\alpha$-synuclein
Canis lupus familiaris $\alpha$-synuclein
Gallus gallus $\alpha$-synuclein
Known IREs in human genes

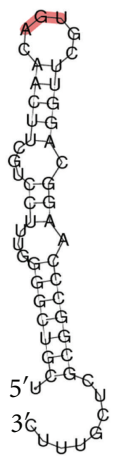

Homo sapiens

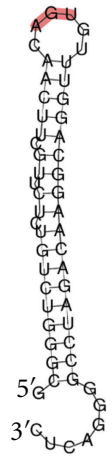

Homo sapiens Homo sapiens

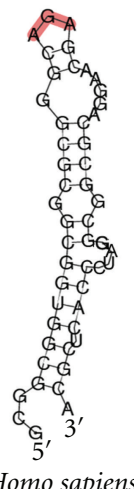

APP
Comparison of human synuclein homologs

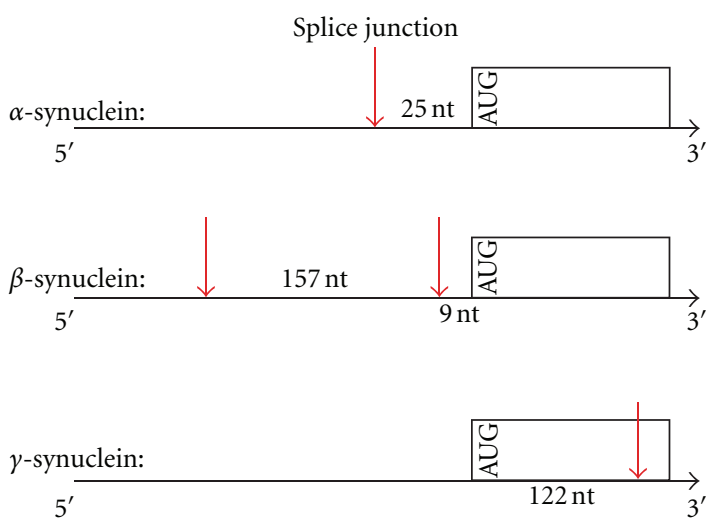

Ferritin H-Chain Ferritin L-Chain

(b)

FIgURe 1: Continued. 
Quantified homologies across the splice junction

\begin{tabular}{|l|r|r|}
\hline \multicolumn{3}{|c|}{ Quantified homology against Homo sapiens } \\
\hline Species & Exon 1 (\%) & Exon 2 (\%) \\
\hline Pan troglodytes & 100 & 100 \\
\hline Mus musculus & 42.3 & 61.5 \\
\hline Rattus norvegicus & 23.1 & 65.4 \\
\hline Bos taurus & 46.2 & 96.2 \\
\hline Canis lupus familiaris & 42.3 & 92.3 \\
\hline Gallus gallus & 34.6 & 69.2 \\
\hline
\end{tabular}

\begin{tabular}{|l|l|}
\hline \multicolumn{2}{|l|}{ Quantified IRE control homology against Homo sapiens } \\
\hline Controls & Homology (\%) \\
\hline L-Ferritin & 31.5 \\
\hline H-Ferritin & 38.9 \\
\hline APP & 31.5 \\
\hline
\end{tabular}

(c)

Figure 1: The 5'untranslated region (5'UTR) of the Parkinson's disease alpha-synuclein (SNCA) transcript is homologous to the ironresponsive element (IRE) in H-ferritin mRNA. (a) Top panel: The SNCA 5'UTR is encoded by exon-1 and exon-2 of the SNCA gene, which can be alternatively spliced to generate either a shorter exon-1/-2 transcript or a longer exon- $1^{\prime}-2$ transcript [58]. Bottom panel: evolutionary alignment of the SNCA 5'UTR relative to the human sequence and the CAGUGN loop/splice site sequences [33]. We quantified the homology across the SNCA splice junction in the 5' untranslated region. (b) RNA/FOLD computer program predictions of RNA stem loops from SNCA 5'UTR sequences $(\triangle G=53 \mathrm{kcal} / \mathrm{mol})$. The human $S N C A$ stem loop resembles the classical IRE RNA stem loop (5'CAGUGN3' loop motif) that controls iron-dependent L- and H-ferritin translation and transferrin receptor (TfR) mRNA stability. Stem loops from the 5'UTRs of several species were predicted to be folded, as described in the Materials and Methods section, and the pseudotriloop AGU is depicted in red lettering at the apex of the H-ferritin IRE [59] where the analogous AGA from the APP IRE is depicted [50]. The human SNCA mRNA exhibited an AGU triloop, whereas, in lower vertebrates, this AGU motif was located in the stem regions of these transcripts. Shown are the arrangement of splice sites and 5'UTR structures in the SNCA, SNCB, SNCG mRNAs. Tabulated: the first table shows the homology between the IRE-like domains in each exon of the human alpha-synuclein compared to its counterparts in different mammalian species. The second table shows the homology between the 5' untranslated regions of SNCA with the APP and ferritin L- and H-chains. This data underscored our identification of cotranslational repression of APP and alpha-synuclein by small molecules, such as phenserine and posiphen.

posiphen, from Microsource Discovery Systems, Inc, was screened at a concentration of $2 \mathrm{uM}$ in triplicate for inhibition of luciferase expression using (APP cells) and (SNCA cells). A compound was scored as a hit if all replicates gave $>65 \%$ inhibition in this assay, and as contradictory if at least one, but not all replicates gave $>65 \%$ inhibition. In order to rule out compounds that reduced luciferase expression due to toxicity, the entire library was also screened in both cell lines for cytotoxicity, using alamar blue (Biosource, Inc.) as the readout. Percent inhibition in the alamar Blue assay was calculated and compared with percent inhibition of luciferase activity. Posiphen and phenserine were among the compounds for which the difference was greater than 40 and which scored as a hit or contradictory in the luciferase assay.

2.6. Transient Transfection Assays. After $24 \mathrm{hr}$ transfection of SH-SY5Y cells in a $100 \mathrm{~mm}$ dish with either (i) SNCA5'UTR-pGL3 or (ii) pGL3 parental vector, cells were then passaged into 6 wells and tested with posiphen and phenserine $(15 \mu \mathrm{M}=$ for $48 \mathrm{hr})$ as shown in Figures 3 and 4 for which dose-response assays were also conducted (not shown) $[34,50]$.

2.7. Luciferase Assay. Cells were plated at 2000 cells/well (APP) or 4000 cells/well (SNCA) in a 384-well white flatbottom plate (Greiner) in a volume of $50 \mathrm{uL}$ of media. Following overnight incubation, $50 \mathrm{~nL}$ of $5 \mu \mathrm{M}$ in DMSO were added using a VPN Pintool. Cells were returned to the incubator of $48 \mathrm{~h}$, then assayed for luciferase activity. Plates were allowed to equilibrate to room temperature. After addition of $25 \mathrm{uL}$ Steady-Glo reagent (Promega), plates were vortexed for $30 \mathrm{sec}$ and 35 minutes later luminescence read on an Infinite F2000 plate reader (Tecan) $[34,50]$.

2.8. Western Blot Assay. Human SH-SY5Y cells and primary cortical neurons (E18 fetal cells) were exposed to increasing concentrations of phenserine and posiphen for 48 hours. Cytoplasmic protein lysates were prepared by homogenizing the cells in midRIPA buffer (25 mM Tris $\mathrm{pH} 7.4,1 \%$ NP40, $0.5 \%$ sodium deoxycholate, $15 \mathrm{mM} \mathrm{NaCl}$, protease inhibitors, RNase inhibitor, and $10 \mu \mathrm{M}$ DTT). Western Blotting for alpha-synuclein was performed using mouse monoclonal anti-alpha-synuclein (BD Transduction Laboratories) and anti-beta-actin (Chemicon). The blots were developed using chemiluminescence (PIERCE) and visualized with a PhosphoImager (BioRad, Hercules, CA), and bands were quantified using QuantityOne software (BioRad).

\section{Results and Discussion}

In Figure 1, we performed a full bioinformatic analysis of the SNCA 5'UTR demonstrating by computer-mediated predictions [50] that the 5'UTR of the SNCA transcript folds into a unique RNA stem loop resembling an iron-responsive element (IRE) RNA structure that is related to, but distinct from, the H-ferritin and APP 5'UTR-specific IREs [33]. We are currently testing the capacity of intracellular iron chelation with desferrioxamine to repress neural $\alpha$-synuclein translation acting via the IRE in the $5^{\prime} \mathrm{UTR}$ of its transcript, as we reported for the APP and ferritin mRNAs $[42,51]$.

Previously, the RNA-directed anticholinesterase drug phenserine, together with its cholinergically inert chiral $(+)$ 
H-Ferritin IRE Loop

$\begin{array}{llllll}\text { H-chain } & 5^{\prime} & 53 & \text { CGGGGTTTCCTGCTTCAACAGT GCTTGGACGGAACCCGGCGCTCGT } & 3^{\prime} & 99 \\ \text { L-chain } & 5^{\prime} & 21 & \text { GTCTGTCTCTTGCTTCAACAGT GT TTGGACGGAAAGATCCGGGGA } & 3^{\prime} & 67 \\ \text { ASYN } & 5^{\prime} & 1 & \text { GGAGTGGCCATTCGACGACAGT GT GGTGTAAAGGAATCATTAGCC } & 3^{\prime} & 50 \\ \text { APP } & 5^{\prime} & 64 & \text { CGGCGGTGGCGGCGCGGGCAGAGCAAGGACGCGGCGGATCCCACTC } & 3^{\prime} & 103\end{array}$

CAGAGCAAGGACG

(a)

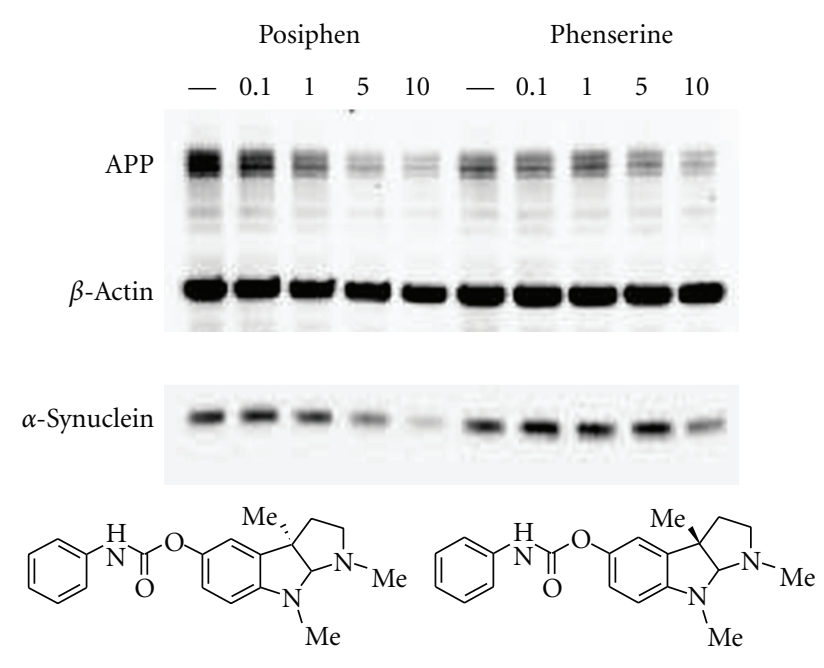

Posiphen ((+)-phenserine)

Phenserine ((-)-phenserine)

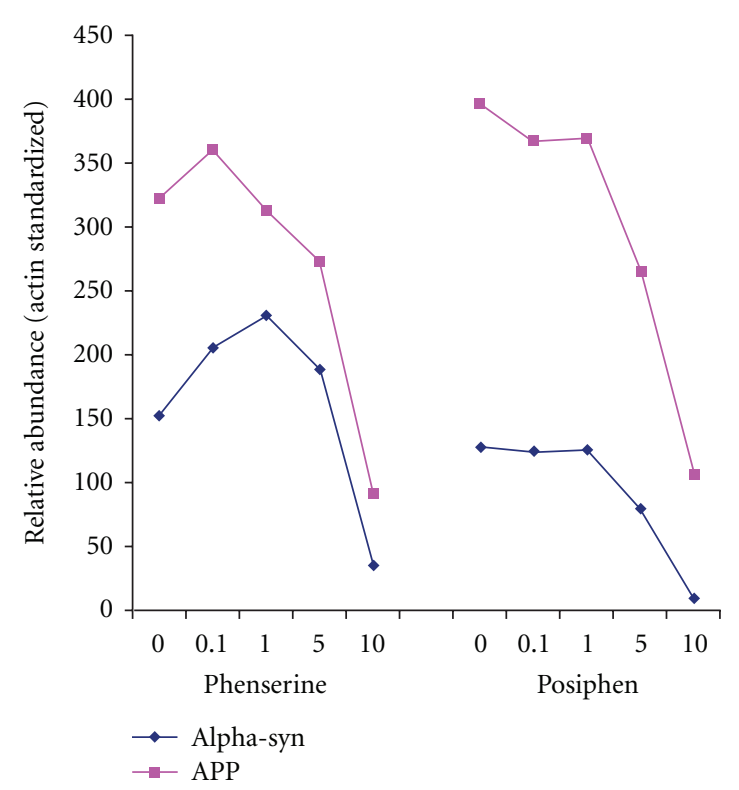

(b)

Figure 2: Posiphen and phenserine decrease both APP and $\alpha$-synuclein levels dose-dependently in dopaminergic SH-SY5Y cells. Panel A: the 5'UTRs of both the APP and SNCA genes are 50\% homologous with the IRE H-ferritin mRNA. Panel B: SH-SY5Y cells were treated with concentrations ranging from 0 to $10 \mu \mathrm{M}$ phenserine and posiphen for $48 \mathrm{~h}$. Harvested cell lysates were prepared. Quantitative Western Blotting established the anti- $\alpha$-syn efficacy of posiphen and phenserine $\left(\mathrm{IC}_{50}<5 \mu \mathrm{M}\right.$ ); after standardization for $\beta$-actin (Densitometry of multiple lanes $(n=8)$ by ImageQuant). Cell viability was unaffected (measured by standardized ATP levels/cell (Tm (Cell-Titer-glo, Promega, Inc.))).

enantiomer, posiphen, which are both in clinical development for $\mathrm{AD}$, was shown to therapeutically limit brain $\mathrm{A} \beta$ levels in wild-type and $\mathrm{AD}$ mouse models [46]. This action was mediated, in whole or in part, by lowering the rate of synthesis of APP, from which $A \beta$ is proteolytically cleaved. Here, we sought to demonstrate that phenserine and posiphen, likewise, blocked $\alpha$-synuclein expression via their related $5^{\prime}$ UTRs, encoding variant versions of the ironresponsive element RNA elements that potentially bind ironregulatory proteins.

To elucidate whether our defined target would translate across species, we provide the results of an evolutionary evaluation of the conservation of IRE RNA stem loops in SNCA mRNA as shown in Figure 1. This is the RNA secondary structure sequence, together with the APP $5^{\prime} \mathrm{UTR}$, that is targeted by phenserine and posiphen, as shown in Figures 2-4. The alpha-synuclein-specific IRE stem loop was formed at the splice junction of the first two exons in $S N C A$ gene [35]. We also compared the predicted structure of the SNCA mRNA IRE with the canonical IREs in the Hferritin and APP transcripts, which are transcribed from the single first exon of their genes confirming the uniqueness of translational repression of SNCA mRNA via its $5^{\prime}$ UTR.

The anticholinesterase phenserine and its $(+)$ enantiomer, posiphen, are proven APP 5'UTR mediated drugs with known pharmacokinetics in rodents and humans and identified target concentrations [46, 52]. Since we anticipated that both agents would be active in limiting $\alpha$-synuclein translation via its $5^{\prime} \mathrm{UTR}$, we had spiked an FDA library with posiphen and phenserine when we formerly screened against the SNCA 5'UTR RNA target [34]. Here, we confirm that both posiphen and phenserine repressed the SNCA 5'UTRdriven translation of a luciferase reporter in stable cells lines. Their capacity to inhibit SNCA mRNA translation is similar 


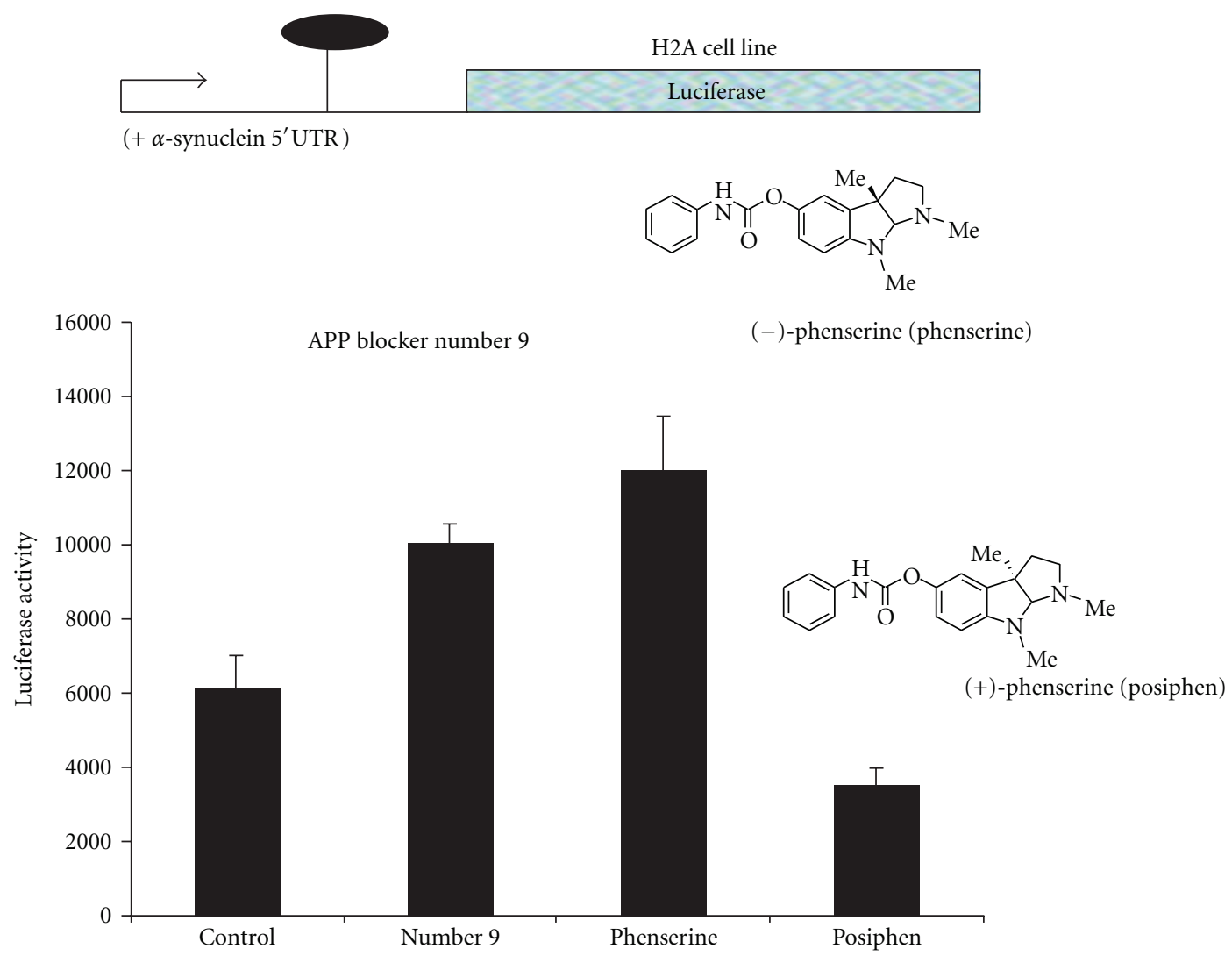

FIGURe 3: Posiphen is a stereospecific inhibitor of alpha synuclein $5^{\prime} \mathrm{UTR}$-directed translation. Transient transfection assays with the SNCA$5^{\prime}$ UTR-pGL3 construct (H2A cell line). Posiphen was a highly selective inhibitor $(10 \mu \mathrm{M})$ of $\alpha$-synuclein $5^{\prime} \mathrm{UTR}$-driven expression of a luciferase reporter gene. This stereoisomer of phenserine inhibited $\alpha$-synuclein $5^{\prime}$ UTR-driven luciferase expression in neural cells (SNCA $5^{\prime}$ UTR-positive transfected neural cells $(N=7)$ ). By contrast, phenserine and the known APP-specific translation number 9 blocker did not suppress alpha synuclein 5'UTR-conferred translation in H2A cells. Phenserine and APP blocker number 9 increased SNCA 5'UTRconferred translation. These data support the mechanism-of-action of posiphen as a highly selective blocker of alpha synuclein $5^{\prime} \mathrm{UTR}$ activity, whereas phenserine (same chemical structure but stereoisomer of posiphen) was previously shown selectively inhibiting translation driven by the APP 5'UTR.

to that of certain other defined FDA drug leads, including three glycosides and an immunosuppressant, mycophenolic acid (secondary Fe chelator), as we previously reported [34].

In Figure 2(a), the $5^{\prime}$ UTRs of both APP and SNCA showed $40 \%$ homology to each other and also 50\% homology to the IRE in $\mathrm{H}$-ferritin mRNA. Multiple Western blot experiments were hence conducted to determine the impact of phenserine compared to posiphen to limit $\alpha$-syn compared to APP expression. In this regard, SH-SY5Y cells were treated with both compounds for 48 hours with concentrations ranging from 0 to $10 \mu \mathrm{M}$. Viability studies determined that this range was well tolerated, in accordance with prior studies. In general and as shown in Figure 2(b), posiphen (in addition to but potentially slightly more potently than phenserine) decreased levels of $\alpha$-syn in a dose-dependent manner in cultured neural cells (SH-SY5Y) as previously reported for APP. Whether this higher potency would translate to primary neurons and in vivo is a focus of future studies. In this paper, this was achieved with a preliminary determined $\mathrm{IC}_{50}$ in the order of $5 \mu \mathrm{M}$, in the absence of toxicity.

Multiple transient transfections of SH-SY5Y cells were performed with the constructs that either translated luciferase driven by the 48 base SNCA $5^{\prime}$ UTR (SNCA$5^{\prime}$ UTR-pGL3) or the empty pGL3 expression vector (Figures 3 and 4). As shown (Figures 3 and 4), posiphen 50\% repressed SNCA $5^{\prime}$ UTR-conferred translation of a luciferase reporter transcript. In this regard, posiphen proved a highly selective inhibitor of SNCA $5^{\prime}$ UTR driven activity since this chirally pure compound inhibited SNCA $5^{\prime}$ UTR-driven luciferase expression in H2A neural cells (i.e., SNCA 5'UTRpositive stably transfected neural cells). By contrast, phenserine and the known APP translation blocker, compound number 9 (included as a comparator), did not suppress 
H2A cell line

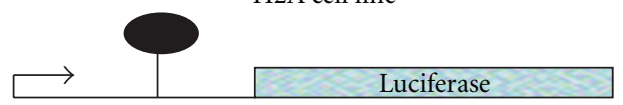

$\left(+\alpha\right.$-synuclein $\left.5^{\prime} \mathrm{UTR}\right)$

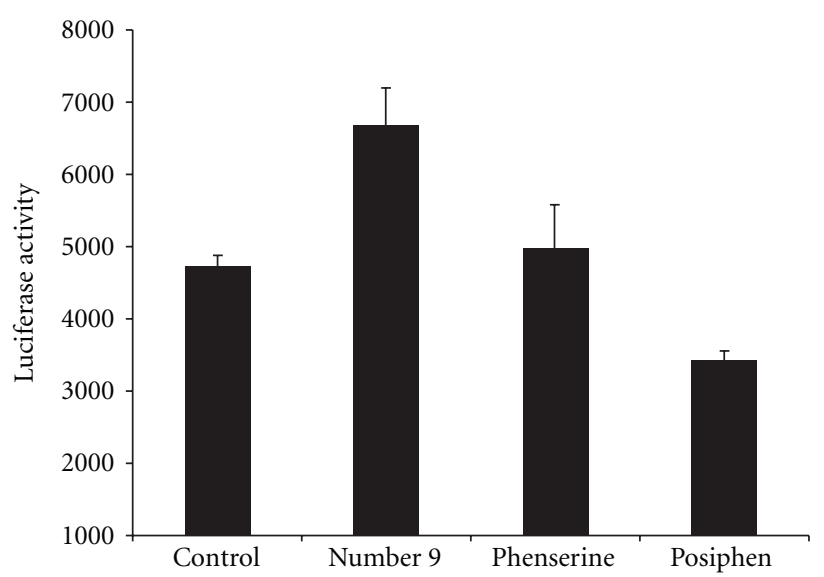

H4-pGL3 cell line

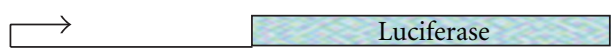

(- $\alpha$-synuclein $\left.5^{\prime} \mathrm{UTR}\right)$

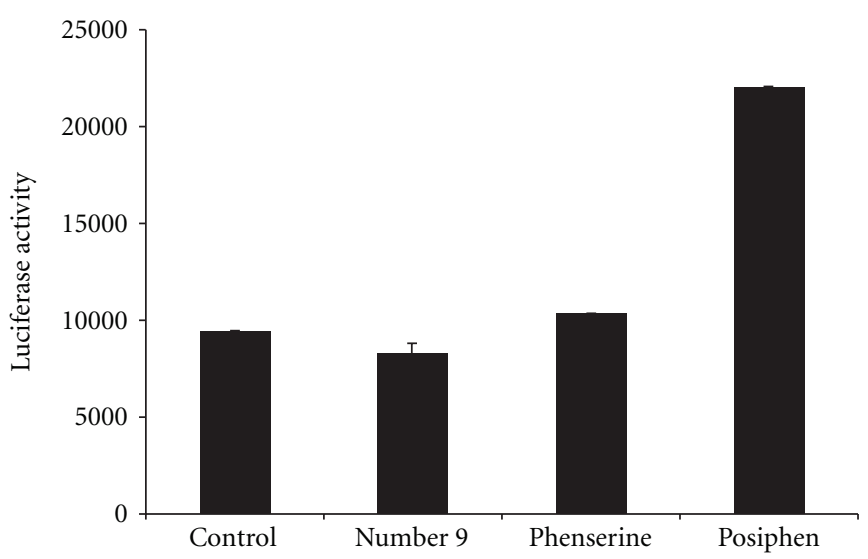

FIGURE 4: Selectivity of posiphen to inhibit translation driven by alpha-synuclein 5'UTR sequences: a second set of transient transfection experiments in which posiphen $(10 \mu \mathrm{M})$ selectively inhibited alpha-synuclein 5'UTR-conferred luciferase expression in SH-SY5Y neural cells (SNCA 5'UTR-positive transfectants, $(N=6)$ ). Confirming selectivity, posiphen increased luciferase expression in pGL3-transfected SHSY5Y cells (** pGL3-SH-SY5Y serves as an experimental control since these cells were transfected with pGL3, which is the same as the H2A construct but lacks the $\alpha$-synuclein 5'UTR).

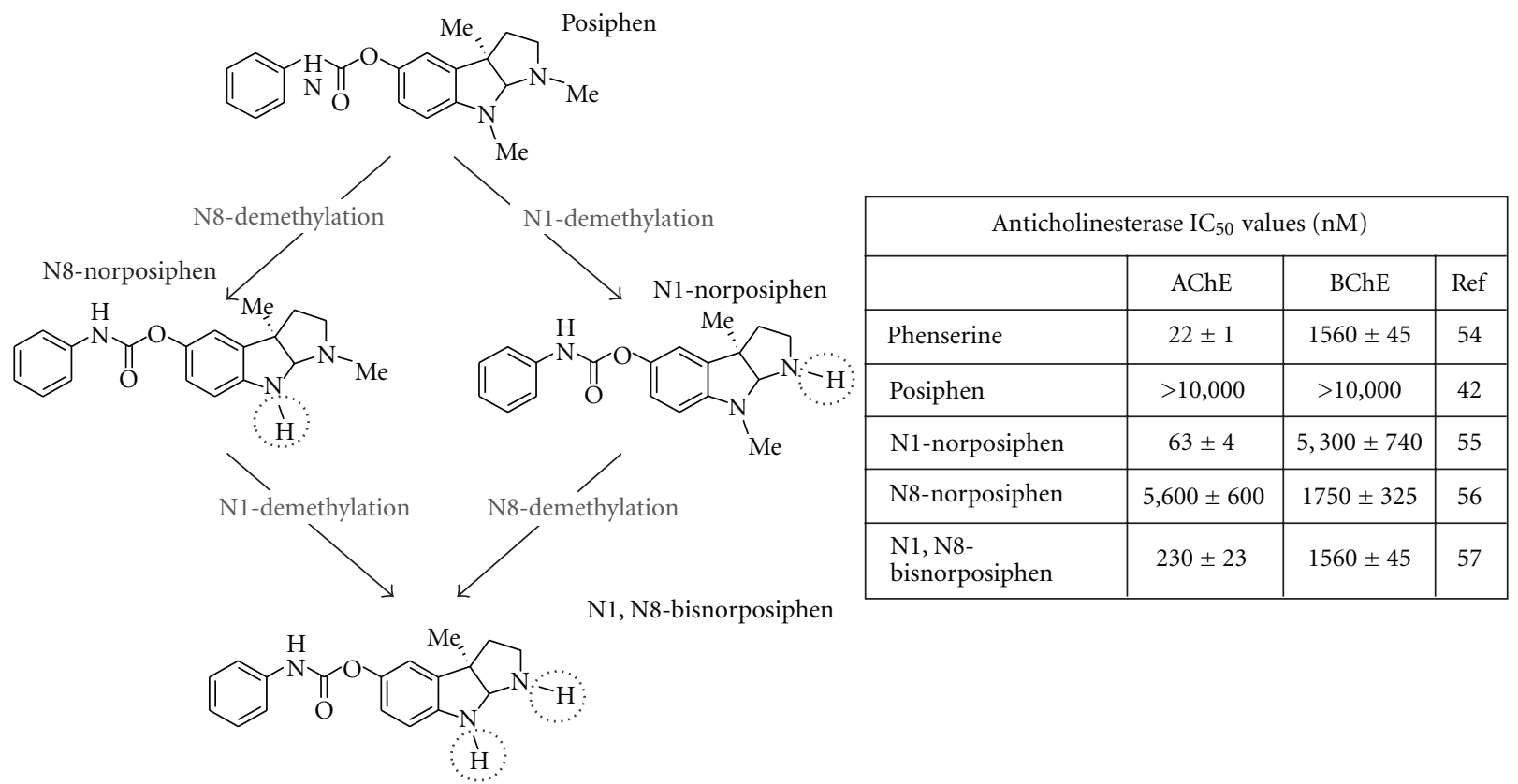

Figure 5: Metabolic analogs of posiphen and their respective anticholinesterase activities [60]. Posiphen is devoid of anticholinesterase activity. However, its phase 1 metabolites, N8 demethylated, N1 demethylated, and di-demethylated N1, N8-bisnorposiphen showed ex vivo AChE and BChE inhibitory activity of clinical relevance $[61,62]$. The compound N8-bisnorposiphen demonstrated no AChE activity. This activity has proven to be dose limiting in human safety studies. 


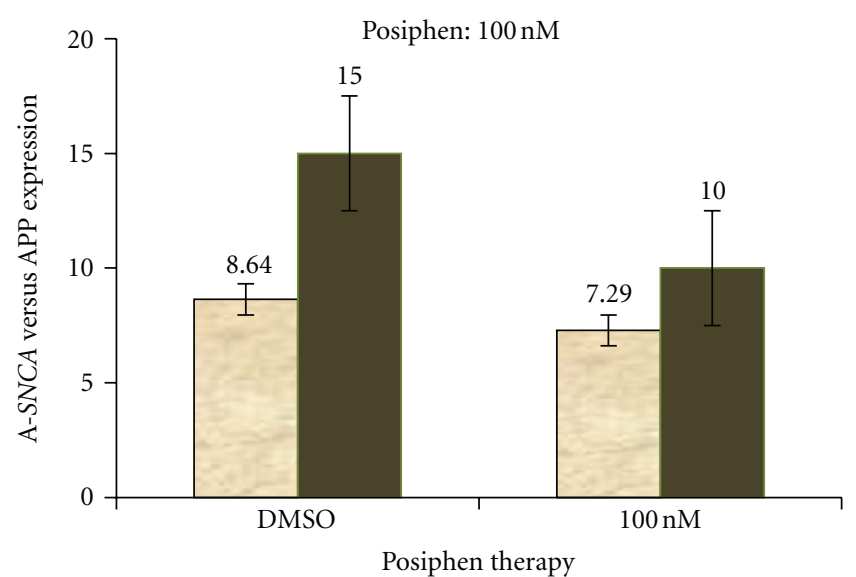

A-SNCA

APP

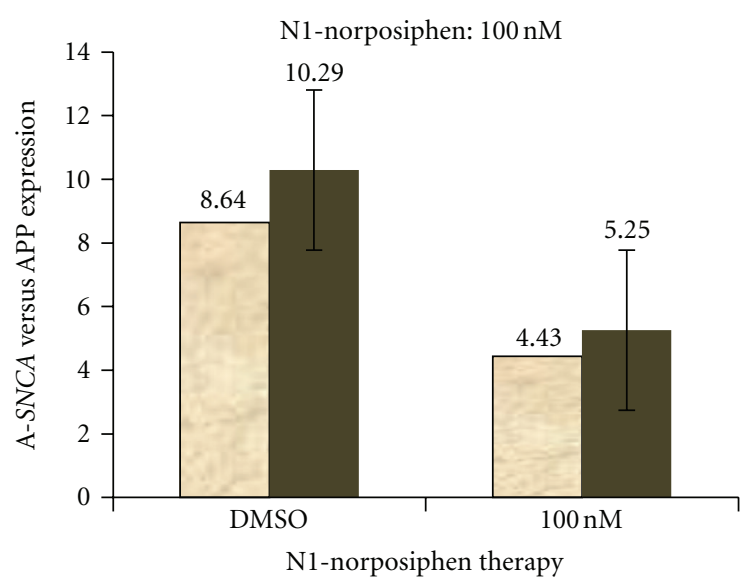

A-SNCA

APP

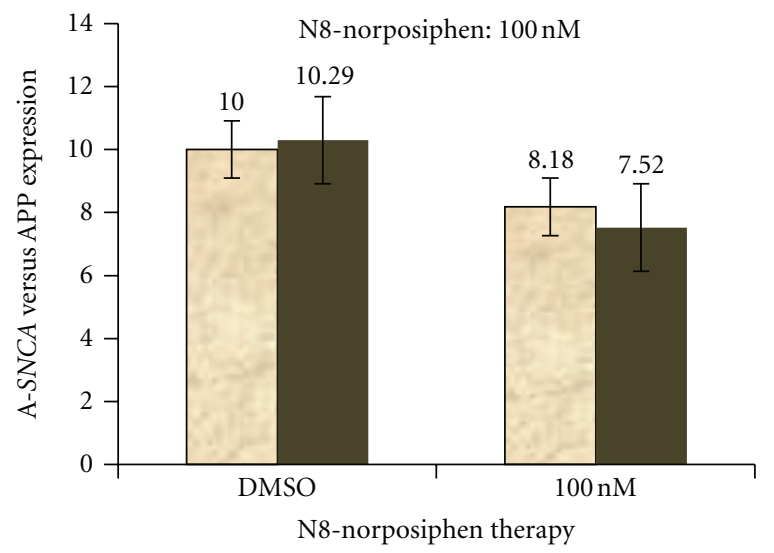

A-SNCA

APP
N1, N8-bisnorposiphen: $100 \mathrm{nM}$

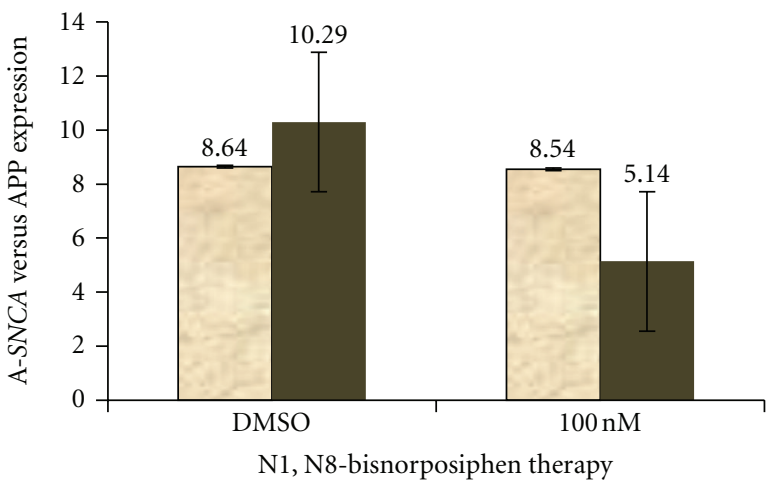

A-SNCA

APP

FIGURE 6: Percent inhibition of $\alpha$-synuclein/APP by posiphen compared with its metabolic analogs in primary E18 neurons (from PAC$\operatorname{Tg}(S N C A)$ mice). Densitometry and quantitation of the relative levels $\alpha$-synuclein/APP from western blots ( $\beta$-actin standardized) after treating primary neurons with posiphen and each of its three metabolic analogs at $100 \mathrm{nM}$ concentration for 48 hours.

SNCA 5'UTR conferred translation in H2A cells. Indeed, phenserine and compound number 9 elevated SNCA $5^{\prime}$ UTRconferred translation.

These data support the mechanism-of-action of posiphen as a highly selective blocker of SNCA 5'UTR activity. However, phenserine-with the identical chemical structure but in a different three-dimensional (chiral) configurationthat has previously been shown to effectively inhibit translation driven by the APP $5^{\prime}$ UTR clearly has different actions to posiphen at the SNCA $5^{\prime} \mathrm{UTR}$. From this, we can deduce that the element of the SNCA 5'UTR targeted by posiphen has a stereospecific component. Additionally, since phenserine lowers $\alpha$-syn levels (Figure 2(b)), further SNCA
RNA sequences are likely involved in controlling this pathway of $\alpha$-syn translational regulation.

Extending beyond transformed neuronal cell lines, primary neurons from wild-type mice and PAC SNCA transgenic mice (PAC-Tg SNCA) were evaluated for the capacity of posiphen to repress $\alpha$-syn expression, as shown in Figure 6 (tested at $100 \mathrm{nM}$ drug concentration). Posiphen proved not only active and well tolerated in SH-SY5Y cells but consistently reduced human $\alpha$-synuclein expression in primary neurons (E18) at doses as low as $1 \mathrm{uM}$ (75\% reduction, not shown) without toxicity. This margin appeared to be greater than its capacity to lower APP production (20\%) (utilized as a positive control) in these same cells (data not shown). 

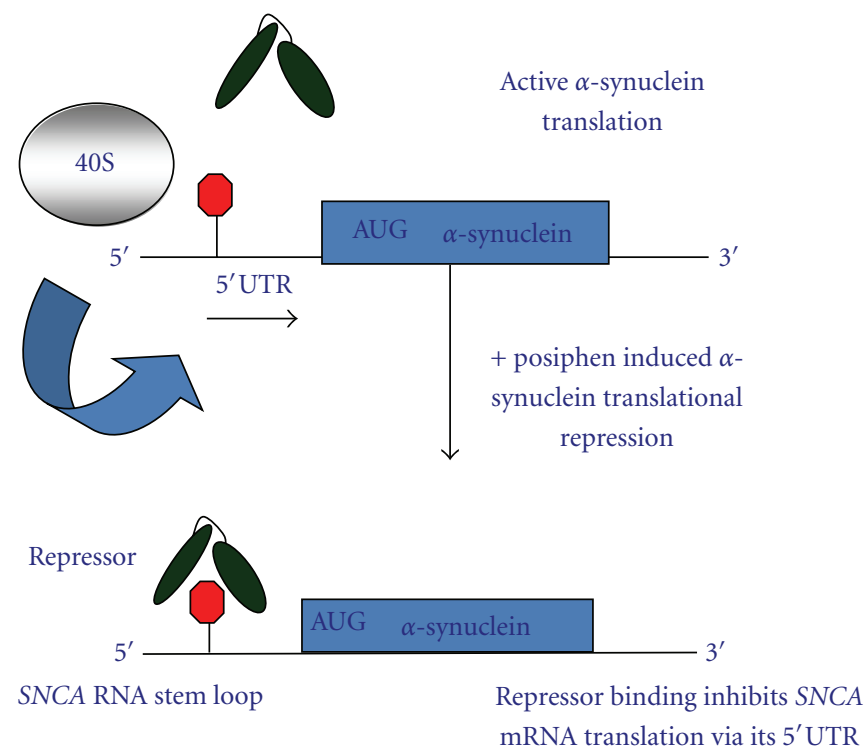

Posiphen enhances repressor binding to the SNCA 5'UTR.

Phenserine acts via other SNCA mRNA sequences

FIGURE 7: A model for translation repression of alpha-synuclein in which its transcript interacts with an RNA repressor protein. In the classical translation repression model, Iron-regulatory protein-1 (IRP1) controls iron dependent expression of the ferritin L- and H-chains. By analogy, phenserine, posiphen and its metabolic analogs would be predicted to increase the repressor as an RNA binding protein to interact with the SNCA 5'UTR after drug treatment. This should prohibit 40 S ribosome access to the $5^{\prime}$ UTR of SNCA mRNA to lower translation.

Following oral administration of posiphen to rodents, dogs, and human, the compound is subjected to metabolic processes and generates the same metabolic profile across these species. Specifically, via a phase 1 metabolism, posiphen undergoes $\mathrm{N}$-demethylation in both the N1 and N8 positions to generate the respective primary metabolites, N1norposiphen and N8-norposiphen (Figure 5). Each then undergoes further $\mathrm{N}$-demethylation to generate the common metabolite, N1, N8-bisnorposiphen. Unlike phenserine, posiphen is devoid of cholinesterase inhibitory activity and, therefore, can be advantageously administered at higher clinical doses (in the order of 5, to 8-fold greater).

As shown in Figure 5, specific N-demethylated metabolites (in particular the N1-nor and N1,N8-bisnorposiphen) possess potentially clinically relevant $\mathrm{IC}_{50}$ values to inhibit acetylcholinesterase (AChE). Such activity is less than phenserine and would be expected to have a slow onset in line with the time-dependent metabolism of posiphen to generate its metabolites. However, with regard to actions on $\alpha$-synuclein expression, activity of the metabolites at this target, where posiphen is potent, could usefully increase and extend the drug's in vivo efficacy. To elucidate this and aid planning for future animal studies, we characterized the relative capacities of posiphen's metabolic analogs to impact $\alpha$-syn expression (Figure 6).

Figure 6 shows a Western Blot analysis representative of three experiments that compared the efficacy of posiphen with that of its three primary metabolites to limit $\alpha$ synuclein expression ex vivo using E18 primary cortical neurons from human SNCA PAC mice. We consistently measured that N1-norposiphen (possessing AChE activity) proved most potent to limit $\alpha$-synuclein expression (by $50 \%$ ), and, likewise, APP expression was also reduced. As assessed at $100 \mathrm{nM}$ in Figure 6, the other metabolites proved to be less active at these targets, indicating that relatively small structural changes (i.e., the loss of a methyl moiety) have significant impact. An assessment of dose-response for each metabolite is a focus of future studies, particularly within the achievable clinical range of the agents in human and in vivo range in animal models (Dr. Maria Maccecchini and Dr. Robert Nussbaum, personal communications).

\section{Conclusions}

We previously reported that phenserine, a (-)-physostigmine analogue and anticholinesterase that reached phase III clinical assessment for AD [53-55], inhibited APP translation though its $5^{\prime}$ UTR $[56,57]$. Phenserine was found to be dose limiting consequent to its AChE inhibitory action, causing the classical cholinergic action of tremors in animal models and nausea in humans. Posiphen, the chirally pure $(+)$ enantiomer of phenserine, by contrast possesses no direct anticholinesterase activity and, likewise, repressed neural 
AD-specific APP translation via its $5^{\prime} \mathrm{UTR}$ in mice. It has recently been developed ( $\mathrm{QR}$ Pharma, Berwyn, PA) to the clinic as an APP synthesis inhibitor in AD to lower both brain $\mathrm{A} \beta$ generation as well as the levels of other toxic proteolytic products of APP. With a very different pharmacological and pharmacokinetic profile to phenserine, it has recently completed single-and multiple-dose escalating phase I clinical assessment in humans, appearing well-tolerated, and a proof of mechanism study, indicating target engagement.

In this paper, we demonstrated in human immortal neuronal cultures, and then ex vivo in primary cortical neurons from a human SNCA genomic mouse model, that posiphen acts as a safe $5^{\prime} \mathrm{UTR}$-directed inhibitor of toxic $\alpha$-synuclein buildup. A final validation of the SNCA $5^{\prime}$ UTR target is to be achieved by unchanged $\beta$-synuclein $(\beta$-syn) and $\gamma$ synuclein ( $\gamma$-syn) expression and is a focus of current studies. Importantly, we determined that key primary metabolites of posiphen, likewise, lower $\alpha$-syn expression and may hence add to posiphen's actions on this target in animal and human studies.

Of further interest, we established that phenserine similarly effectively reduced $\alpha$-syn levels but achieves this via a different mechanism, compared to posiphen, potentially by interacting through other elements in the $5^{\prime}$ UTR or even 3'UTR, and thereby highlighting the sensitivity of the target to small structure-activity changes. These studies additionally demonstrate how $\alpha$-synuclein and Aß pathomechanisms can converge via interaction of the two amyloidogenic proteins [38-40], although $A ß$ and $\alpha$-syn rarely co-localize in amyloid deposits [41], but provide evidence that a drug targeting one disease may have therapeutic potential in another.

In this regard, we noted that there is $50 \%$ sequence similarity between the $5^{\prime}$ UTRs of the APP and SNCA genes. We therefore predicted overlap in the spectrum of drugs that would suppress APP mRNA translation through its $5^{\prime}$ UTR with those that suppress $\alpha$-synuclein, highlighting in particular phenserine and posiphen (with preliminary $\mathrm{IC}_{50}$ values in the order of $5 \mu \mathrm{M}$ and less). In future studies, we aim to characterize the anti- $\alpha$-synuclein efficacy of posiphen analogs in addition to metabolic analogs and other compounds in primary neurons and establish the in vivo efficacy of the most effective SNCA 5'UTR translation blockers.

\section{Acknowledgments}

The authors thank Prof. Robert Nussbaum and Dr. YienMing Kuo (UCSF) for their PAC-Tg(SNCA) mice. This research was supported (i) for J. T. Rogers by the Michael J. Fox Foundation Novel Drug Discoveries Award, National Institute of Aging R01 AG20181, NINDS R21NS059434, Alzheimer's Association Zenith Award 09-131352 and ISOA (ii) for N. H. Greig by the Intramural Research Program, National Institute on Aging, National Institutes of Health, (iii) for I. Cantuti-Caselvetri: the MGH/MIT Morris Udall Center of Excellence in PD Research (NIH NS38372) and the APDA Advanced Center for Parkinson Research at MGH, and (iv) for C. M. Cahill by NIH HD012437 29 S-1.

\section{References}

[1] G. S. Standaert and A. B. Young, "Treatment of the central nervous system degenerative disorders," in Goodman \& Gilaman's The Pharmacological Basis of Therapeutics, L. L. Brunton, Ed., pp. 527-545, McGraw-Hill, New York, NY, USA, 2005.

[2] M. R. DeLong and J. L. Juncos, "Parkinson's disease and other movement disorders," in Harrison's Neurology in Clinical Medicine, S. L. Hauser, Ed., pp. 295-314, McGraw-Hill, New York, NY, USA, 2006.

[3] L. S. Forno, "Neuropathology of Parkinson's disease," Journal of Neuropathology and Experimental Neurology, vol. 55, no. 3, pp. 259-272, 1996.

[4] I. Cantuti-Castelvetri and D. G. Standaert, "Neuroprotective strategies for Parkinson's disease," Current Neuropharmacology, vol. 2, pp. 153-168, 2004.

[5] K. R. Chaudhuri and Y. Naidu, "Early Parkinson's disease and non-motor issues," Journal of Neurology, vol. 255, no. 5, pp. 33-38, 2008.

[6] K. R. Chaudhuri and P. Odin, "The challenge of non-motor symptoms in Parkinson's disease," Progress in Brain Research, vol. 184, pp. 325-341, 2010.

[7] K. R. Chaudhuri and A. H. Schapira, "Non-motor symptoms of Parkinson's disease: dopaminergic pathophysiology and treatment," The Lancet Neurology, vol. 8, no. 5, pp. 464-474, 2009.

[8] K. A. Jellinger, "Synuclein deposition and non-motor symptoms in Parkinson disease," Journal of the Neurological Sciences, vol. 310, no. 1, pp. 107-111, 2011.

[9] D. O. Claassen, K. A. Josephs, J. E. Ahlskog, M. H. Silber, M. Tippmann-Peikert, and B. F. Boeve, "REM sleep behavior disorder preceding other aspects of synucleinopathies by up to half a century," Neurology, vol. 75, no. 6, pp. 494-499, 2010.

[10] R. Postuma and J. F. Gagnon, "Cognition and olfaction in Parkinson's disease," Brain, vol. 133, no. 12, p. e160, 2010, author reply e161.

[11] M. A. Hely, W. G. J. Reid, M. A. Adena, G. M. Halliday, and J. G. L. Morris, “The Sydney Multicenter Study of Parkinson's disease: the inevitability of dementia at 20 years," Movement Disorders, vol. 23, no. 6, pp. 837-844, 2008.

[12] W. G. J. Reid, M. A. Hely, J. G. L. Morris et al., "A longitudinal study of Parkinson's disease: clinical and neuropsychological correlates of dementia," Journal of Clinical Neuroscience, vol. 3 , no. 4, pp. 327-333, 1996.

[13] A. Larner, "Cholinesterase inhibitors: beyond Alzheimer's disease," Expert Review of Neurotherapeutics, vol. 10, no. 11, pp. 1699-1705, 2010.

[14] J. T. Olin, D. Aarsland, and X. Meng, "Rivastigmine in the treatment of dementia associated with Parkinson's disease: effects on activities of daily living," Dementia and Geriatric Cognitive Disorders, vol. 29, no. 6, pp. 510-515, 2010.

[15] F. A. Schmitt, D. Aarsland, K. S. Brønnick, Xiangyi Meng, S. Tekin, and J. T. Olin, "Evaluating rivastigmine in mildto-moderate Parkinsons disease dementia using ADAS-cog items," American Journal of Alzheimer's Disease and other Dementias, vol. 25, no. 5, pp. 407-413, 2010.

[16] F. A. Schmitt, M. R. Farlow, X. Meng, S. Tekin, and J. T. Olin, "Efficacy of rivastigmine on executive function in patients with parkinson's disease dementia," CNS Neuroscience \& Therapeutics, vol. 16, no. 6, pp. 330-336, 2010.

[17] D. J. Burn, "The treatment of cognitive impairment associated with parkinson's disease," Brain Pathology, vol. 20, no. 3, pp. 672-678, 2010. 
[18] J. T. Greenamyre and T. G. Hastings, "Parkinson's-divergent causes convergent mechanisms," Science, vol. 304, no. 5674, pp. 1120-1122, 2004.

[19] K. Gwinn-Hardy, "Genetics of parkinsonism," Movement Disorders, vol. 17, no. 4, pp. 645-656, 2002.

[20] D. F. Clayton and J. M. George, "The synucleins: a family of proteins involved in synaptic function, plasticity, neurodegeneration and disease," Trends in Neurosciences, vol. 21, no. 6, pp. 249-254, 1998.

[21] M. H. Polymeropoulos, C. Lavedan, E. Leroy et al., "Mutation in the $\alpha$-synuclein gene identified in families with Parkinson's disease," Science, vol. 276, no. 5321, pp. 2045-2047, 1997.

[22] M. H. Polymeropoulos, "Autosomal dominant Parkinson's disease and alpha-synuclein," Annals of Neurology, vol. 44, no. 3, supplement 1, pp. S63-S64, 1998.

[23] H. Braak, K. Del Tredici, U. Rüb, R. A. I. De Vos, E. N. H. Jansen Steur, and E. Braak, "Staging of brain pathology related to sporadic Parkinson's disease," Neurobiology of Aging, vol. 24, no. 2, pp. 197-211, 2003.

[24] C. M. Cahill and J. T. Rogers, "Interleukin (IL) $1 \beta$ induction of IL-6 is mediated by a novel phosphatidylinositol 3-kinasedependent $\mathrm{AKT} / \mathrm{I} \kappa \mathrm{B}$ kinase $\alpha$ pathway targeting activator protein-1," Journal of Biological Chemistry, vol. 283, no. 38, pp. 25900-25912, 2008.

[25] B. Wolozin and N. Golts, "Iron and Parkinson's disease," Neuroscientist, vol. 8, no. 1, pp. 22-32, 2002.

[26] S. Gal, M. Fridkin, T. Amit, H. Zheng, and M. B. H. Youdim, "M30, a novel multifunctional neuroprotective drug with potent iron chelating and brain selective monoamine oxidase-ab inhibitory activity for Parkinson's disease," Journal of Neural Transmission, Supplement, no. 70, pp. 447-456, 2006.

[27] C. M. Cahill, D. K. Lahiri, X. Huang, and J. T. Rogers, "Amyloid precursor protein and alpha synuclein translation, implications for iron and inflammation in neurodegenerative diseases," Biochimica et Biophysica Acta, vol. 1790, no. 7, pp. 615-628, 2009.

[28] J. P. Taylor, I. F. Mata, and M. J. Farrer, "LRRK2: a common pathway for parkinsonism, pathogenesis and prevention?" Trends in Molecular Medicine, vol. 12, no. 2, pp. 76-82, 2006.

[29] A. E. Oakley, J. F. Collingwood, J. Dobson et al., "Individual dopaminergic neurons show raised iron levels in Parkinson disease," Neurology, vol. 68, no. 21, pp. 1820-1825, 2007.

[30] O. Weinreb, T. Amit, S. Mandel, and M. B. H. Youdim, "Neuroprotective molecular mechanisms of (-)-epigallocatechin-3gallate: a reflective outcome of its antioxidant, iron chelating and neuritogenic properties," Genes \& Nutrition, vol. 4, no. 4, pp. 283-296, 2009.

[31] I. Cantuti-Castelvetri, J. Klucken, M. Ingelsson et al., "Alphasynuclein and chaperones in dementia with Lewy bodies," Journal of Neuropathology and Experimental Neurology, vol. 64, no. 12, pp. 1058-1066, 2005.

[32] L. Reznichenko, T. Amit, H. Zheng et al., "Reduction of ironregulated amyloid precursor protein and $\beta$-amyloid peptide by (-)-epigallocatechin-3-gallate in cell cultures: implications for iron chelation in Alzheimer's disease," Journal of Neurochemistry, vol. 97, no. 2, pp. 527-536, 2006.

[33] A. L. Friedlich, R. E. Tanzi, and J. T. Rogers, "The 5'untranslated region of Parkinson's disease $\alpha$-synuclein messengerRNA contains a predicted iron responsive element [1]," Molecular Psychiatry, vol. 12, no. 3, pp. 222-223, 2007.

[34] J. T. Rogers, S. Mikkilineni, I. Cantuti-Castelvetri et al., "The alpha-synuclein 5/untranslated region targeted translation blockers: anti-alpha synuclein efficacy of cardiac glycosides and Posiphen," Journal of Neural Transmission, vol. 118, no. 3, pp. 493-507, 2011.

[35] D. Olivares, X. Huang, L. Branden, N. H. Greig, and J. T. Rogers, "Physiological and pathological role of alpha-synuclein in parkinson's disease through iron mediated oxidative stress; the role of a putative iron-responsive element," International Journal of Molecular Sciences, vol. 10, no. 3, pp. 12261260, 2009.

[36] K. Ueda, H. Fukushima, E. Masliah et al., "Molecular cloning of cDNA encoding an unrecognized component of amyloid in Alzheimer disease," Proceedings of the National Academy of Sciences of the United States of America, vol. 90, no. 23, pp. 11282-11286, 1993.

[37] V. M. Y. Lee, B. I. Giasson, and J. Q. Trojanowski, "More than just two peas in a pod: common amyloidogenic properties of tau and $\alpha$-synuclein in neurodegenerative diseases," Trends in Neurosciences, vol. 27, no. 3, pp. 129-134, 2004.

[38] P. H. Jensen, P. Hojrup, H. Hager et al., "Binding of Abeta to alpha- and beta-synucleins: identification of segments in alpha-synuclein/NAC precursor that bind Abeta and NAC," Biochemical Journal, vol. 323, no. 2, pp. 539-546, 1997.

[39] K. A. Conway, J. D. Harper, and P. T. Lansbury, "Accelerated in vitro fibril formation by a mutant $\alpha$-synuclein linked to earlyonset Parkinson disease," Nature Medicine, vol. 4, no. 11, pp. 1318-1320, 1998.

[40] Han Hogyu, P. H. Weinreb, and P. T. Lansbury Jr., "The core Alzheimer's peptide NAC forms amyloid fibrils which seed and are seeded by beta-amyloid: is NAC a common trigger or target in neurodegenerative disease?" Chemistry \& Biology, vol. 2, no. 3, pp. 163-169, 1995.

[41] J. Q. Trojanowski and V. M. Y. Lee, “Parkinson's disease and related neurodegenerative synucleinopathies linked to progressive accumulations of synuclein aggregates in brain," Parkinsonism \& Related Disorders, vol. 7, no. 3, pp. 247-251, 2001.

[42] J. T. Rogers, J. D. Randall, C. M. Cahill et al., "An ironresponsive element type II in the $5^{\prime}$-untranslated region of the Alzheimer's amyloid precursor protein transcript," Journal of Biological Chemistry, vol. 277, no. 47, pp. 45518-45528, 2002.

[43] J. A. Duce, A. Tsatsanis, M. A. Cater et al., "Iron-Export Ferroxidase Activity of $\beta$-Amyloid Precursor Protein is Inhibited by Zinc in Alzheimer's Disease," Cell, vol. 142, no. 6, pp. 857867, 2010.

[44] P. Davies, D. Moualla, and D. R. Brown, "Alpha-synuclein is a cellular ferrireductase," PLOS ONE, vol. 6, no. 1, Article ID e15814, 2011.

[45] P. Davies, X. Wang, C. J. Sarell et al., "The synucleins are a family of redox-active copper binding proteins," Biochemistry, vol. 50, no. 1, pp. 37-47, 2011.

[46] D. K. Lahiri, D. Chen, B. Maloney et al., "The experimental Alzheimer's Disease drug posiphen $[(+)$-phenserine] lowers amyloid- $\beta$ peptide levels in cell culture and mice," Journal of Pharmacology and Experimental Therapeutics, vol. 320, no. 1, pp. 386-396, 2007.

[47] I. Nakano and A. Hirano, "Parkinson's disease: neuron loss in the nucleus basalis without concomitant Alzheimer's disease," Annals of Neurology, vol. 15, no. 5, pp. 415-418, 1984.

[48] Y. M. Kuo, Z. Li, Y. Jiao et al., "Extensive enteric nervous system abnormalities in mice transgenic for artificial chromosomes containing Parkinson disease-associated $\alpha$-synuclein gene mutations precede central nervous system changes," $\mathrm{Hu}$ man Molecular Genetics, vol. 19, no. 9, Article ID ddq038, pp. 1633-1650, 2010.

[49] B. Ray, J. A. Bailey, S. Sarkar, and D. K. Lahiri, "Molecular and immunocytochemical characterization of primary neuronal 
cultures from adult rat brain: differential expression of neuronal and glial protein markers," Journal of Neuroscience Methods, vol. 184, no. 2, pp. 294-302, 2009.

[50] H. H. Cho, C. M. Cahill, C. R. Vanderburg et al., "Selective translational control of the Alzheimer amyloid precursor protein transcript by iron regulatory protein-1," Journal of Biological Chemistry, vol. 285, no. 41, pp. 31217-31232, 2010.

[51] S. Bandyopadhyay, X. Huang, H. Cho, N. H. Greig, M. B. Youdim, and J. T. Rogers, "Metal specificity of an ironresponsive element in Alzheimer's APP mRNA 5' untranslated region, tolerance of $\mathrm{SH}-\mathrm{SY} 5 \mathrm{Y}$ and $\mathrm{H} 4$ neural cells to desferrioxamine, clioquinol, VK-28, and a piperazine chelator," Journal of Neural Transmission, Supplement, no. 71, pp. 237247, 2006.

[52] M. Maccecchini, "Posiphen's pharmacokinetics and mecha883nism of action in mild cognitive impaired patients," Alzheimer's \& Dementia, vol. 6, no. 4, supplement 1, p. e54, 2010.

[53] B. Winblad, E. Giacobini, L. Frölich et al., "Phenserine efficacy in Alzheimer's disease," Journal of Alzheimer's Disease, vol. 22, no. 4, pp. 1201-1208, 2010.

[54] N. H. Greig, K. Sambamurti, Q. S. Yu, A. Brossi, G. B. Bruinsma, and D. K. Lahiri, "An overview of phenserine tartrate, a novel acetylcholinesterase inhibitor for the treatment of Alzheimer's disease," Current Alzheimer Research, vol. 2, no. 3, pp. 281-290, 2005.

[55] J. Klein, "Phenserine," Expert Opinion on Investigational Drugs, vol. 16, no. 7, pp. 1087-1097, 2007.

[56] K. T. Y. Shaw, T. Utsuki, J. Rogers et al., "Phenserine regulates translation of $\beta$-amyloid precursor protein mRNA by a putative interleukin-1 responsive element, a target for drug development," Proceedings of the National Academy of Sciences of the United States of America, vol. 98, no. 13, pp. 7605-7610, 2001.

[57] A. Venti, T. Giordano, P. Eder et al., "Integrated role of desferrioxamine and phenserine targeted to an iron-responsive element in the APP-mRNA 5' -untranslated region," Annals of the New York Academy of Sciences, vol. 1035, pp. 34-48, 2004.

[58] Y. Xia, T. Saitoh, K. Uéda et al., "Characterization of the human $\alpha$-synuclein gene: genomic structure, transcription start site, promoter region and polymorphisms," Journal of Alzheimer's Disease, vol. 3, no. 5, pp. 485-494, 2001.

[59] J. B. Goforth, S. A. Anderson, C. P. Nizzi, and R. S. Eisenstein, "Multiple determinants within iron-responsive elements dictate iron regulatory protein binding and regulatory hierarchy," RNA, vol. 16, no. 1, pp. 154-169, 2010.

[60] W. Luo, Q. S. Yu, M. Zhan et al., "Novel anticholinesterases based on the molecular skeletons of furobenzofuran and methanobenzodioxepine," Journal of Medicinal Chemistry, vol. 48, no. 4, pp. 986-994, 2005.

[61] Q. S. Yu, X. F. Pei, H. W. Holloway, N. H. Greig, and A. Brossi, "Total syntheses and anticholinesterase activities of (3aS)-N(8)-norphysostigmine, (3aS)-N(8)-norphenserine, their antipodal isomers, and other $\mathrm{N}(8)$-substituted analogues," Journal of Medicinal Chemistry, vol. 40, no. 18, pp. 2895-2901, 1997.

[62] Q. S. Yu, N. H. Greig, H. W. Holloway, and A. Brossi, "Syntheses and anticholinesterase activities of (3aS)-N1,N8bisnorphenserine, (3aS)-N1,N8-bisnorphysostigmine, their antipodal isomers, and other potential metabolites of phenserine," Journal of Medicinal Chemistry, vol. 41, no. 13, pp. 23712379, 1998 . 


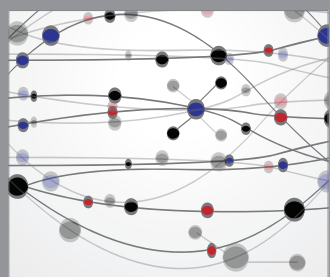

The Scientific World Journal
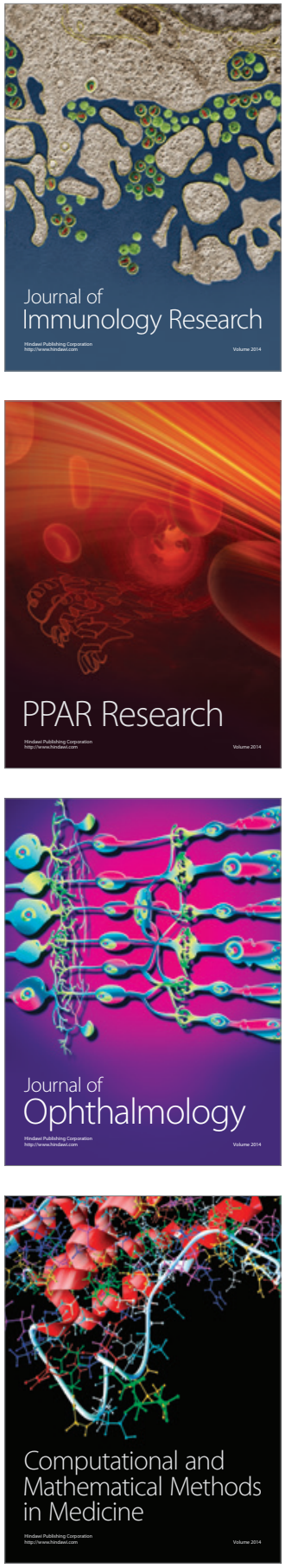

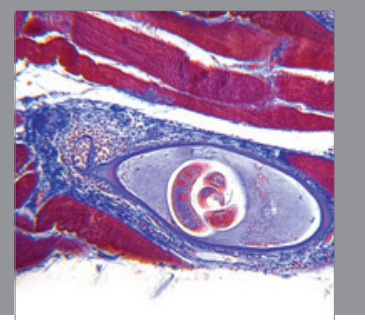

Gastroenterology

Research and Practice
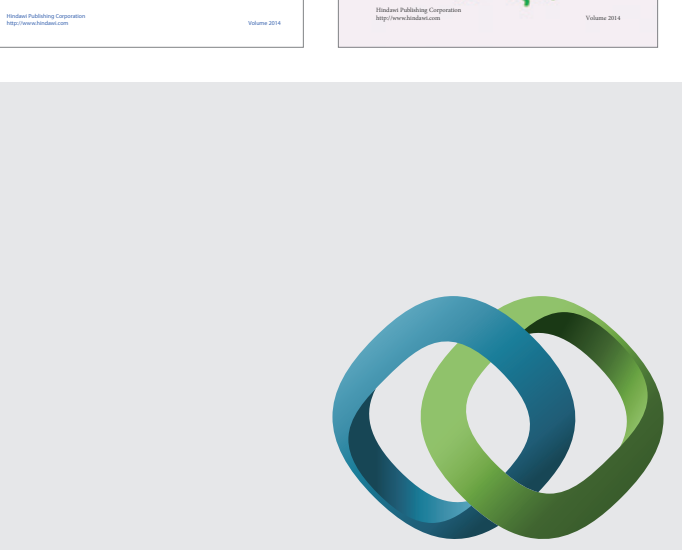

\section{Hindawi}

Submit your manuscripts at

http://www.hindawi.com
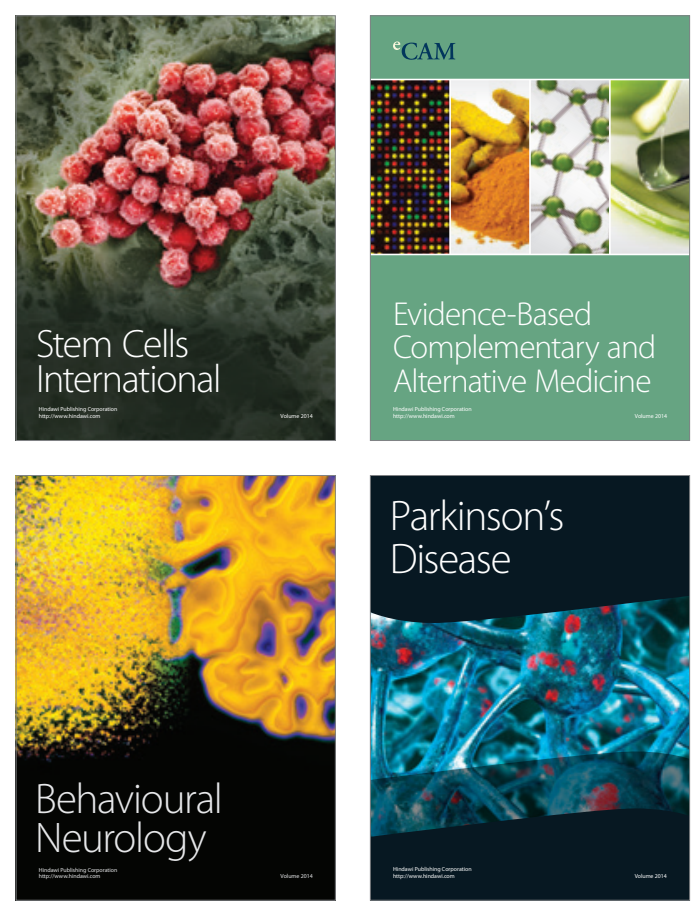

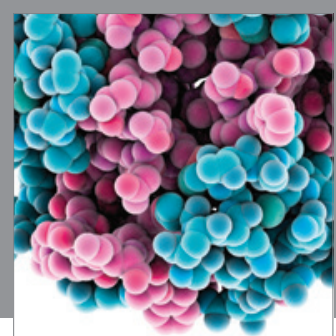

Journal of
Diabetes Research

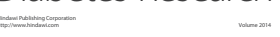

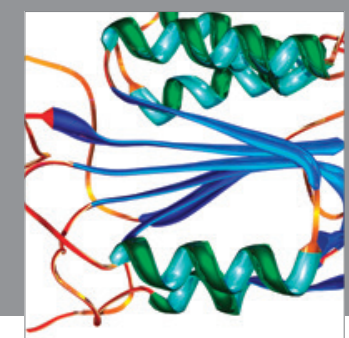

Disease Markers
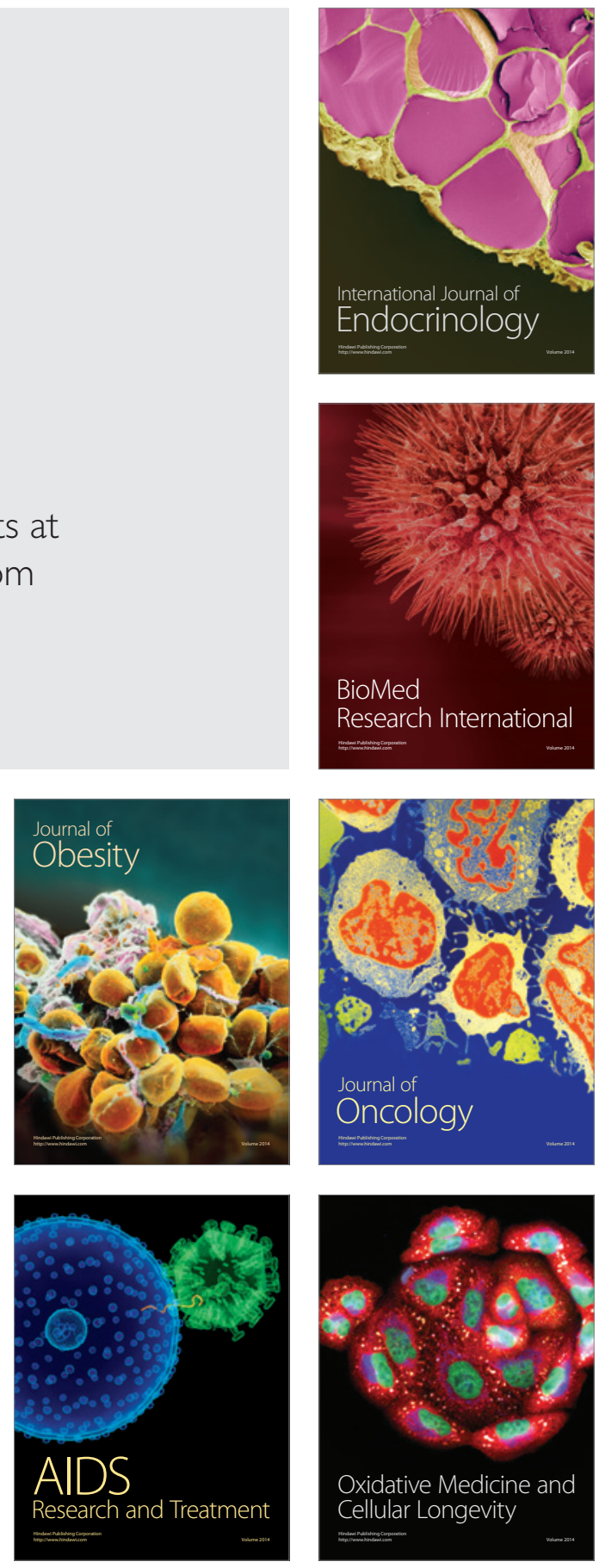